\title{
MesSAge BASED ANALysis ON SignAling System NUMBER 7 AND ITS COMPARISON WITH MULTI- FREQUENCY CODED SIGNALING
}

\author{
Md. Shah Alam ${ }^{1}$ and Mohammad Rezaul Huque $\mathrm{Khan}^{2 *}$ \\ Dept. of Electronics and Telecommunication Engineering, Daffodil International University, Bangladesh \\ E-mail: 1shah_alam@daffodilvarsity.edu.bd,2rhkcu@yahoo.com
}

\begin{abstract}
A message based comparative study between signaling system \#7(SS7) and MFC Signaling is done. Here the limitations of SS7 and MFC signaling are also studied. The reasons for the transition from Multi-frequency Coded (MFC) Signaling to SS7 has been discussed.
\end{abstract}

Keywords: Common Channel Signaling No.7 (CCS7), R2 Signaling or Multi-frequency Coded (MFC) Signalin and, SS7 message.

\section{Introduction}

The over increasing demand of telecommunication in the world wide significantly involves telecommunication signaling systems. The Common Channel Signaling no.7 is usually termed as Signaling System No.7 (SS7). The purpose of this paper is to study the signaling systems R2 or MFC, SS7 [1], to find the limitations of the above signaling system, analysis of the signaling systems (R2 and SS7) on the basis of their message format. An overall comparison between the two systems has been studied. This paper also focuses on the transition of MFC to SS7.

\section{Common Channel Signaling No. 7}

Common Channel Signaling System No. 7 (i.e., SS7 or C7) is a global standard for telecommunications defined by the International Telecommunication Union (ITU) Telecommunication Standardization Sector (ITU-T). The standard defines the procedures and protocol by which network elements in the Public Switched Telephone Network (PSTN) exchange information over a digital signaling network to effect wireless (cellular) and wireline call setup, routing and control [2, 3, 4]. The SS7 network and protocol are used for: (i) Basic call setup, management, and tear down.

(ii) Wireless services such as Personal Communications Services (PCS), wireless roaming, and mobile subscriber authentication.

(iii) Local Number Portability (LNP).

(iv) Toll-free (800/888) and toll (900) wireline services.

(v) Enhanced call features such as call waiting, call forwarding, calling party name/number display/ restriction/rejection, and three-way calling.

(vi) Interaction with Network Databases and Service Control Points (SCP) for service control.

(vii) Handling congestion and priorities.

(viii) Efficient and secure worldwide telecommunications.

SS7 started to make inroads in the $1980^{\text {ec }} \mathrm{s}$ because it was a major technological advance. It was a fully digital technology running at the then blazing speed of 64,000 bps (versus no more than $30 \mathrm{bps}$ for tone-based signaling). Messages which had previously been limited to a few digits in length could now be over 200 bytes long. Signaling messages were no longer transmitted „in-band (within the voice circuit), so they could be exchanged with network elements that did not have voice trunks, allowing the development of services such as 1-800, the intelligent network (IN) [5] and wireless mobility management.

\section{R2 Signaling}

R2 signaling was known originally as MFC signaling [3]. It was developed cooperatively by European telecommunication equipment manufacturers and the European conference of 
postal and telecommunications administrations (usually represented as CEPT), and was introduced in the 1960s. It is still used in many national networks in Europe, Latin America, Australia, and Asia. Although R2 signaling has been defined in ITU-T Q.400-Q.490 recommendations [6], there are many variations in how R2 is implemented. The R2 signaling is an international signaling system that transmits numerical and other information relating to the called and the calling subscribers' lines. There are two elements of R2 Signaling. They are Line Signaling (supervisory signals) and Inter-register Signaling (call setup/control signals) [7,8].

\subsection{Line Signaling}

R2 line signaling is a family of protocols which govern the resource acquisition and resource release related to a two-party telephone call attempt and, if successful, the establishment of a two-party telephone call. Although in the 1960s R2 line signaling was represented as electrical pulses on a two-wire or four-wire circuit, by the latter 1970 s these analog electrical pulses also could be represented in digital form by a signaling DS0 (usually known as frame format of American first-order digital multiplex) in the trunk, which is normally channel 16 in an E1(a frame contains 32 eight-bit time slots defined by CEPT) trunk [9].

\subsection{Register Signaling}

R2 register signaling is a family of protocols which govern the conveyance of addressing information during the addressing phase and how the call attempt turned out during the disposition phase. Although in the 1960s R2 register signaling was represented by electromechanical devices which could generate multi-frequency audio tones and by electromechanical devices which could detect those audio tones, by the latter 1970s these electromechanical registers also could be represented by digitized Pulse Code Modulation (PCM) audio in DS0 channels of an E1 other than the R2 line signaling DS0 in that E1 [10].

\section{Limitations}

\subsection{Limitations of R2 Signaling}

Some of the major limitations of R2 signaling are (i) much slower than common channel signaling. (ii) R2 signaling cannot be used on Time Assignment Speech Interpolation (TASI) equipped trunks. (iii) It is more costly to interface the MF registers and line signaling hardware for the individual trunks used in R2 signaling. (iv)The transfer of additional signaling information for processing a call is not possible in case of R2 signaling. So MFC or R2 signaling does not provide the requirements to meet the new and future challenges [3].

\subsection{Limitations of SS7 Signaling}

Some of the major restrictions with SS7 are: (i) Link speed and capacity, message size, addressing, international routing [12]. (ii) The size of a single data packet must be less than about 250 bytes. (iii) Lack of seamless international operation, unlike the TCP/IP protocol used on the internet and the basic addressing method, the point code, stops at a country boundary, etc [13].

\section{Message Based Analysis of the Signaling System (R2 \& Ss7)}

A message based analysis on SS7 signaling system and R2 signaling system is presented here. Usually SS7 mainly deals with ISUP signaling i.e., Integrated Services user part signaling. There are some basic differences between these two systems (R2 and SS7) in their message format and how the signaling is done. An SS7 message format (ISUP TRACE) [14] and an R2 message format (R2 TRACE) [14] is given below for example. From the analysis of ISUP traces it is shown that there are three types of codes included in ISUP message format. These are originating point code (OPC), destination point code (DPC), and circuit identification code (CIC) and they are represented in hexadecimal number. The CIC identifies a trunk within a trunk group. The CIC field has a length of 12 bits, and thus can identify trunks in groups of up to 4095 trunks. When an ISUP message is sent or received, it contains these codes. But in case of R2 messages it contains only the destination point code. For this reason a R2 signal message does not contain its caller ID. In case of R2 message it is also observed that it conveys two types of signal, one is forward signal (FS) and another is backward signal (BS). On the other hand ISUP message 
consists of initial address message (IAM), subsequent address messages (SAM), address complete message (ACM), answer message (ANM), release message (REL), release complete message (RLC), and many other message parts. The different parts of the message contain different types of signaling information (SI).

The transfer of additional signaling information for processing a call is most necessary. This is possible only in case of SS7. In ISUP message, many signaling information just like call origination, trunk type, call start time, end time and the release of call is also mentioned in the message. In case of R2 signaling the transfer of additional signaling information is not provided.

\subsection{An SS7 Message Format: ISUP TRACE}

TRC-ISUP: RTE 700;

M8480 ISUP SIGNALING TRACE

RESULT $=\mathrm{OK}$

ITEM ASP TI LNK TRK S_TRK E_TRK

RTE

RTE CNT TRC_TYPE

700100 ALL

M8480 ISUP SIGNALING TRACE (1/100)

ITEM ASP TI LNK TRK S_TRK E_TRK

RTE $0 * * * * * * * 34 * * * * * * * *$

RTE CALL S_TIME E_TIME TRC_TYPE

700 OGT 13:43:34 13:43:40 ALL

OPC DPC CIC

NAT/H“e OcOf NAT/H’0001 H’0002

MSG DIR DATA

I AM 006000 0F 0302000403100300

SAM 0200028000

SAM 0200028002

SAM 0200028002

ACM 160400

REL 0200028090
RLC 00

REL_REASON $=$ NORMAL CALL CLEARING

\subsection{An R2 Message Format}

R2 TRACE

TRC-R2: RTE=601;

M8430 R2 SIGNALING TRACE

TRC_TYPE $=$ RTE

RTE $\mathrm{NO}=601$

COUNT $=100$

RESULT $=\mathrm{OK}$

REASON $=$ TRACE RAG OK

M8430 R2 SIGNALING TRACE

TRC_MODE $=$ RTE-ALL TRC_CNT $=001 / 100$

RTE_NO $=0601$ ASP_NO $=00$

TRK_NO $=0130$ R2_NO $=014$

FS CNT $=08$ BS CNT $=08$

$\mathrm{S} / \mathrm{R}=\mathrm{RECEIVER}$ SIG_TIME $=2064 \mathrm{MSEC}$

$\mathrm{FS}=0310101010100204 \mathrm{xx}$ xx xx xx xx

$\mathrm{BS}=0101010101010306 \mathrm{xx}$ xx xx xx xx

\section{Transition from MFC To SS7}

There are several reasons for the move from MFC to SS7.These are faster call setup times (compared to in-band signaling using MF signaling tones). The speed of operation results in reduced post-dialing delays and consequently, this allows the sending of an increased number of signals for additional customer services. Complex messages, instead of simple signals, allow SS7 to offer more services and increased flexibility to meet new and future service requirements. More efficient use of voice circuits, especially on international or long distance calls, where the voice channel is only occupied when the called party is available.

\section{Comparison between SS7 Signaling And R2 Signaling}

A Comparative study between Signaling System No.7 and R2 Signaling is shown below in a tabular form. 

Table 1: Comparison of Signaling System No. 7 and R2 Signaling or MFC Signaling

\begin{tabular}{|c|c|c|}
\hline $\begin{array}{l}\text { Comparative } \\
\text { Features }\end{array}$ & Signaling System No.7 & R2 Signaling or MFC Signaling \\
\hline 1.Channel mode & Common channel signaling is done. & $\begin{array}{l}\text { Channel associated signaling (CAS) is } \\
\text { done. }\end{array}$ \\
\hline $\begin{array}{l}\text { 2.Channel } \\
\text { bandwidth }\end{array}$ & $\begin{array}{l}\text { In SS7, an E1 frame has } 32 * 8=256 \\
\text { bits and bit rate of the system is } \\
8000 * 256=2048 \mathrm{kbps} \text {. }\end{array}$ & $\begin{array}{l}\text { Signaling frequency is } 3825 \mathrm{~Hz} \text { and } 300- \\
3400 \mathrm{~Hz} \text { for subscriber speech. }\end{array}$ \\
\hline $\begin{array}{l}\text { 3.Supporting } \\
\text { trunks }\end{array}$ & $\begin{array}{l}\text { SS7 can be used on Time } \\
\text { Assignment Speech Interpolation } \\
\text { (TASI) equipped trunks. }\end{array}$ & $\begin{array}{l}\text { R2 signaling cannot be used on TASI } \\
\text { equipped trunks. }\end{array}$ \\
\hline $\begin{array}{l}\text { 4.Time slot for } \\
\text { signaling }\end{array}$ & $\begin{array}{l}\text { There is no fixed time slot dedicated } \\
\text { for signaling in SS7. }\end{array}$ & $\begin{array}{l}\text { There is a fixed time slot, TS16 dedicated } \\
\text { for signaling in R2 signaling system. }\end{array}$ \\
\hline $\begin{array}{l}\text { 5.Time slot (TS) } \\
\text { for voice or data } \\
\text { transfer }\end{array}$ & $\begin{array}{l}30 \text { TS is allotted for voice or data } \\
\text { transfer and each TS can be used for } \\
\text { bidirectional i.e., incoming and } \\
\text { outgoing call processing. }\end{array}$ & $\begin{array}{l}\text { First } 15 \text { TS is used for incoming and the } \\
\text { rest of the } 15 \text { are used for outgoing call } \\
\text { processing. }\end{array}$ \\
\hline 6.Interfacing cost & $\begin{array}{l}\text { It is often less costly to interface the } \\
\text { processing equipment of SPC } \\
\text { exchanges with a relatively small } \\
\text { number of signaling links used in } \\
\text { SS7. }\end{array}$ & $\begin{array}{l}\text { It is more costly to interface the MF } \\
\text { registers and line signaling hardware for } \\
\text { the individual trunks used in R2 } \\
\text { signaling. }\end{array}$ \\
\hline 7.Signaling speed & $\begin{array}{l}\text { SS7 is much faster than multi } \\
\text { frequency signaling. }\end{array}$ & $\begin{array}{l}\text { Multi-frequency signaling is much slower } \\
\text { than common channel signaling. }\end{array}$ \\
\hline $\begin{array}{l}\text { 8.Additional } \\
\text { information } \\
\text { transfer }\end{array}$ & $\begin{array}{l}\text { The transfer of additional signaling } \\
\text { information for processing a call is } \\
\text { possible in case of SS7. }\end{array}$ & $\begin{array}{l}\text { The transfer of additional signaling } \\
\text { information for processing a call is not } \\
\text { possible in case of MFC signaling. }\end{array}$ \\
\hline 9.Flexibility & $\begin{array}{l}\text { Common channel signaling messages } \\
\text { provide a more flexible way to } \\
\text { transfer both the classical } \\
\text { supervision and other types of call } \\
\text { control information. }\end{array}$ & $\begin{array}{l}\text { In MFC signaling messages provide no } \\
\text { flexibility like SS7, because the signals } \\
\text { on a trunk necessarily relate to that trunk. }\end{array}$ \\
\hline 10. Caller identity & $\begin{array}{l}\text { In SS7, caller identity and the calling } \\
\text { party category is sent from the } \\
\text { originating to the terminating } \\
\text { exchange. }\end{array}$ & $\begin{array}{l}\text { In CCITT-R2 (International Telephone } \\
\text { and Telegraph Consultative Committee) } \\
\text { signaling, caller identity and the calling } \\
\text { party category is not sent from the } \\
\text { originating to the terminating exchange. }\end{array}$ \\
\hline $\begin{array}{l}\text { 11. Access } \\
\text { capability }\end{array}$ & $\begin{array}{l}\text { Subscriber cannot access the SS7 } \\
\text { signaling links. This avoids the blue- } \\
\text { box fraud problems arise in case of } \\
\text { CAS signaling. }\end{array}$ & $\begin{array}{l}\text { This kind of facility is not available in } \\
\text { MFC signaling. For this reason blue-box } \\
\text { fraud problems arise in CAS which uses } \\
\text { FDM trunk groups. }\end{array}$ \\
\hline $\begin{array}{l}12 \text { Signal } \\
\text { Supervision }\end{array}$ & $\begin{array}{l}\text { SS7 supervision signaling is intended } \\
\text { for both way analog and digital } \\
\text { trunks. }\end{array}$ & $\begin{array}{l}\text { The supervision signaling of CCITT-R2, } \\
\text { intended for one-way analog trunks only } \\
\text { [3]. }\end{array}$ \\
\hline 13. Compatibility & $\begin{array}{l}\text { SS7 has the compatibility to meet the } \\
\text { new and future service requirements. }\end{array}$ & $\begin{array}{l}\text { MFC or R2 signaling does not provide } \\
\text { the requirements to meet the new and } \\
\text { future challenges. }\end{array}$ \\
\hline $\begin{array}{l}\text { 14.Need for } \\
\text { signaling link } \\
(\mathrm{SL})\end{array}$ & $\begin{array}{l}\text { In SS7, a common signaling link } \\
\text { (SL) carries signaling messages for a } \\
\text { number of trunks. }\end{array}$ & $\begin{array}{l}\text { In CAS systems, the signaling } \\
\text { information for a trunk is carried by the } \\
\text { trunk itself. There is no need of signaling } \\
\text { link in R2 signaling. }\end{array}$ \\
\hline
\end{tabular}




\section{Conclusions}

In this paper an effort has been made to give an overview of R2 and SS7signaling. Emphasize is given on the basic limitations of R2 signaling. However, these limitations do not arise in case of SS7. From the analysis some limitations of SS7 in case of link speed and capacity, message size, addressing and international routing have arisen that mentioned in the section 4.2. High speed SS7 links are a good long-term solution to the message size problem, but will not help until the majority of Signaling Transfer Points (STPs) are upgraded to support them. For international signaling, an alternate address method known as global title translation is necessary. But, this is more complex than point codes, and requires management of distinct routing tables in every STP for each global title type.

The standard link speed with SS7 is 64 kbps. The capacity can be increased by implementing up to sixteen SS7 links at a single signaling point. The capacity can be further expanded by implementing 1.5 Mbps links i.e. an entire T1 (a frame format contains 24 eight-bit time slots defined by Bell Laboratories) PCM frame. Theoretically it is possible but practically it is not so easy to done. We should also think about addressing scheme and the routing methodology compatible with different countries.

\section{References}

[1] A history of Engineering and Science in the Bell System, Chapter 12, Bell Telephone Laboratories, Inc., 1982.

[2] http://www.itu.int/ITU-T/

[3] John G. Van Bosse, "Signaling in Telecommunication Networks", Published by Wiley -Interscience. ISBN number 0- 471-57377-9, Chapter 4, pp. 90-106, Chapter 5 pp. 109-124, Chapter 7, pp. 150-158.

[4] http://www.c7.com/ss7/ss7_tutorials.htm

[5] R.K. Berman, J.H. Brewster, "Perspective on the AIN architecture," IEEE Comm. Mag., 31, no.2, February 1992.

[6] Specifications of Signaling Systems R1 and R2, Rec.Q.310-Q.331 (R1 Signaling) and Q.400-Q.480 (R2 Signaling), CCITT Red Book, VI.4, ITU, Geneva, 1985.

[7] S. Welch, Signaling in Telecommunications Networks, Peter Peregrinus Ltd, Stevenage, U.K.,1981.

[8] M. den Hertog, "Interregister Multifrequench Code Signaling for Telephone Switching in Europe", Elec. Comm., 38, 1963.

[9] R.L. Freeman, "Telecommunication System Engineering", Second Edition, Wiley-Inter science, New York, 1989.

[10] Digital Networks, Transmission Systems and Multiplex Equipment, CCITT Red Book, III.3, ITU, Geneva, 1985.

[11] Behrouz A. Forouzan, "Data Communications and Networking", Published by Mc Graw Hill, Third Edition, 2004-2005, Chapter 20, pp. 519-535.

[12] http://www.cnp.wireless.com/articlearchive/wireless20 review/

[13] David Crowe"s „Wireless Telecom" Articles, “2002'Q4: SS7 Now, IP Later?”, May 14, 2007 : Cellular Networking Perspectives Ltd.

[14] http://www.mercurykr.com/ DTS 4000 Operation Manual, ISUP TRACE, Vol.I. pp. 22-30, R2 TRACE, pp. $10-21$. 